\title{
Article
}

\section{Blockchain during COVID-19: The Technology to Help Society}

\author{
Guendalina Capece* (D) and Domitilla Passiatore \\ Department of Enterprise Engineering, University of Rome “Tor Vergata", Via del Politecnico 1, \\ 00133 Rome, Italy; domitillapassiatore@gmail.com \\ * Correspondence: capece@dii.uniroma2.it; Tel.: +39-06-72597801
}

\footnotetext{
check for updates

Citation: Capece, G.; Passiatore, D. Blockchain during COVID-19: The Technology to Help Society. Sustainability 2021, 13, 10478. https:/ / doi.org/10.3390/su131810478
}

Academic Editors: Caterina Tricase, Angela Tarabella, Roberto

Leonardo Rana and Pasquale Giungto

Received: 8 June 2021

Accepted: 9 September 2021

Published: 21 September 2021

Publisher's Note: MDPI stays neutral with regard to jurisdictional claims in published maps and institutional affiliations.

Copyright: (C) 2021 by the authors. Licensee MDPI, Basel, Switzerland. This article is an open access article distributed under the terms and conditions of the Creative Commons Attribution (CC BY) license (https:/ / creativecommons.org/licenses/by/ $4.0 /)$.

\begin{abstract}
At the end of 2019, a new coronavirus was reported in the form of unknown pneumonia: It was the beginning of the SARS-CoV-2 pandemic. It is crucial to develop the strategies to manage this new pandemic in order to improve their effectiveness, as opposed to the ones adopted in 2003 during the SARS pandemic. In 17 years, new digital technologies and tools have been created; therefore, we can take advantage of them in the social management of the pandemic. The aim of our paper is to evaluate how blockchain technology can be used and what advantages it brings in managing an emergency situation such as of the COVID-19 pandemic. In particular, the effects of the adoption of this technology will be assessed when applied both to the management of the information flow between health infrastructures, and to track monetary, technical, and medical supply donations to hospital structures. We therefore propose a high-level, decentralized architecture that assists in administrating large-scale information and stores the gathered data in a blockchain supervised by the authorities. The distrust suffered by institutions today, the fear experienced in the last year due to the pandemic, and the birth of a technology that allows for the creation of reliable networks, pose thoughtful challenges to be faced to recover trust and hope for the future again.
\end{abstract}

Keywords: SARS-CoV-2 pandemic; blockchain technology; monetary donations; sanitary material donations

\section{Introduction}

Pandemics are not unknown to mankind. In fact, there have been many instances in which epidemic waves had to be dealt with, frequently for years at a time. We are currently facing SARS-CoV-2, but it has been since Man began to organize himself in society and create groups of people living together that contagious diseases have played a significant role. As the world population grew larger and diseases spread, affecting various regions of the planet and thus becoming a threat to the population, the need arose to start documenting the first pandemics. These pandemics have often transformed the societies in which they appeared and have almost changed or decisively influenced the course of history.

In December 2019, some cases of pneumonia of unknown etiology were notified to the World Health Organization (WHO) Country Office in China. A few weeks later this unknown pathogen was identified as part of the coronavirus family and named SARS-CoV2. Since then, the novel coronavirus epidemic, caused by the COVID-19 disease, expanded in a few weeks throughout the entire world, with an increasing number of infected people and deaths. According to the observed spread of the infection, it was understood early that there was an urgent need to develop more efficient ways to manage activities in order to contain the disease's potential devastating effects.

In Italy the contagion spread mostly across the northern part of the country. Prompt measures had to be taken in order to deal with what soon became not just a sanitary emergency, but a proper national calamity. Unprecedented measures of austere confinement had to be taken as a means of containing the spread of the virus.

In fact, during the SARS pandemic in 2003, adherence to basic public health and infection-control measures, including contact investigation, infection control at health care 
facilities, and community containment, were found to be crucial strategies to contain the infection. The critical lesson taught by the SARS experience was clearly not forgotten, as shown from the countermeasures adopted in 2019, when Italy went into a two-month lockdown, as did nearly the entire rest of the world. During lockdown all non-essential activities were prohibited. While patients fought for their lives in overloaded hospitals, the rest of the population stayed confined in their own homes, in an atmosphere of fear and disquiet due to the unforeseeable end of the pandemic and its unpredictable dramatic consequences. If prevention is still a better strategy than treatment, we should probably focus on new strategic approaches for pandemic management.

Several implements, both digital and technological, have come out in the past 20 years that have reshaped our lives in ways we could have never had imagined. Some of these could be implemented to advance the traditional tools already being used to contain the spread of SARS-CoV-2, thus increasing their efficiency. Blockchain is one of them.

Since 2008, the year blockchain was created in order to enable the Bitcoin technology, it has found application in different fields. The reason behind its ample use lies in the intrinsic qualities it shares with other distributed ledger technologies: It guarantees a high level of security through the use of cryptography and data inalterability through the use of a consensus mechanism.

With our work we intend to advocate for the use of blockchain technology to facilitate the management of the information flow between health infrastructures and to track monetary, technical, and medical supply donations to hospital structures during a pandemic emergency.

\section{Literature Review}

Nakamoto's white paper in 2008 [1] begun a revolution called Bitcoin and the consequent blockchain-based cryptocurrency innovation. Nakamoto's idea was to create a trusted non-territorial digital currency, not conditioned by centralized and financial institutions [2].

After 2008, blockchain began to polarize much scientific and media attention, besides people's enthusiasms regarding its possible uses and role in decentralizing societies [3] and the consequent independence from central authorities. Indeed, much interest has been drawn to the positive or disruptive changes that an extensive adoption of this technology will bring to our societies. The mainstream of research is focused upon the Bitcoin environment, since Bitcoin is currently the most frequently used and important technology using blockchain, with the largest user base. There are also ongoing studies that challenge the sustainability of Bitcoin, considering the environmental impacts, social issues, and economic aspects of the blockchain-based infrastructure $[4,5]$. Thirteen years after Nakamoto's white paper, blockchain technology passed over the mere purpose in the cryptocurrency field, but until 2017 few papers had explored its promised disruptive potential far beyond IT [6]. It is, in fact, quite remarkable that by the time the COVID-19 pandemic had spread, other than Bitcoin only a handful other applications of blockchain had been exploited.

In the management of the pandemic, blockchain is playing a decisive role, growing as a critical technological answer to the new needs that have arisen from this crisis, such as safe, trackable, and cost-effective means to successful decision-making, and faster intervention. In the field of healthcare, the versatility of blockchain allows for a use that is able to satisfy a variety of demands, from data access to data sharing, granting, at the same time, a sufficient level of security. Other examples consist of blockchain platforms specifically designed for precision medicine and/or clinical trials.

For the last few months blockchain has been, and still is, showing adequate opportunities to become fundamental in fighting against COVID-19 as it allows for efficient tracking and monitoring solutions, guaranteeing a transparent supply chain of vital products and donations and secure payments. Blockchain technology is capable of enhancing the healthcare sector in all the areas particularly affected by the pandemic, including improvements to clinical trials, tracking donations, managing supply-chain operations, etc. Although plenty of answers have been offered to the issues surfaced during the pandemic, many of them 
only remained simple idea suggestions, lacking a concrete evaluation of their effectiveness. Furthermore, the possible uses of blockchain have attracted high expectations because its possible applications are foreseen in almost every human field $[7,8]$.

Despite the growing popularity of blockchain in recent years, since the spread of the COVID-19 pandemic, most of the literature about this technology has still focused on its technological facet, thus neglecting to address the other challenges it poses. Once the technology is consolidated, the consequent question is whether it should be applicable in other equally complex and crucial contexts, such as the management of the pandemic [9-12]. Blockchain could be proven to be especially useful thanks to its intrinsic adaptability to a range of diverse demands, from data sharing, to data security, to data access. Blockchain is quickly becoming a solid technological option in managing the pandemic, as it is able to provide safe, trackable, and cost-effective means that allow the optimal course of action to be chosen efficiently and, hence, action to be taken quickly. By virtue of its effective tracking and monitoring features, today blockchain could be an exceptional asset in the fight against the virus, guaranteeing the transparency of both the supply chain of essential products and of the secure management of donations.

In the following paragraph we proceed to examine some of the papers in which the subject of the use of blockchain applications in the fight against COVID-19 is explored. Within the field of health care, many areas have been impacted by this outbreak that can benefit from the application of blockchain technology. Some of these include the management of clinical trials [13-15] through the direct registration of new data the very moment they are created, with consequent increased data fidelity, boosted data sharing, and guaranteed conformity with the current regulations; an increase in the variety of components of medical supply chains, including, but not limited to, better cost-efficiency, decreased safety hazards in the workplace, and time optimization in the management of the entire process $[16,17]$; a safeguard of user privacy that at the same time allows patients' movements to be kept track of in order to prevent the infringement of social distancing regulations [16]; the collection of data concerning the spread of the virus and its containment in order to study, examine, and compare them [18,19]; real-time updates on the spread of the virus thanks to the analysis of patients' movements [20,21]; and the elimination of many of the downsides of delegating donation management to intermediaries, such as the exploitation of the donations and the extra cost the use of intermediaries entails, therefore boosting social cohesion [22,23]. Should governments adopt such a network, new instruments to develop strategies to combat the virus, such as predictions about the epidemic (with consequent preventive isolation of unaffected areas) and the tracking of its spread would be available, since data introduced in this network would be inherently trustworthy, incorruptible, and transparent [24]. A remarkable initiative is the MiPasa Project: an open platform for attested COVID-19 data built on Hyperledger Fabric that utilizes data analytics and privacy tools that were previously only available to elite financial institutions and adapted them for a public health context. This new data streaming platform uses IBM Blockchain Platform and IBM Cloud. MiPasa draws data from the World Health Organization, the Centers for Disease Control, and the Israeli Public Health Ministry, and any qualified agencies are able to easily integrate new platforms of their own with the simple use of APIs. This will make it vastly easier to collect, organizes, and study information about the outbreak's spread and containment. The main aim of this project is to help public health officials, the business and scientific community, and the public at large to efficiently fight the ongoing COVID-19 pandemic. Because this information is recorded on a permissioned ledger, additional applications can be quickly built on top of it. Indeed, as the world continues trying to manage and contain the ongoing COVID-19 outbreak, officials battling the pandemic need tools and valid information at scale to help foster a greater sense of security for the public [18]. 


\section{The COVID-19 Outbreak}

Coronaviruses are one of the RNA virus groups that cause infection in the respiratory system; they belong to the order of Nidovirales in the Coronaviridae family. This kind of virus is commonly found in camels and bats, as well as other animal species. Occasionally, a process that experts in the field call "species jump" or "cross-species transmission" occurs: As a result of their evolution, these animal viruses can develop the ability to infects humans. Both direct contact between humans and animals (or their meat) and the capability of these viruses to replicate within human hosts, have been identified as the sine qua non of this animal-to-human transmission. This alone, however, does not automatically trigger a human-to-human virus diffusion. In order for this to happen, first the virus has to adapt to the body of the infected subject in order to raise its numbers by replication; second, it has to be able to transmit itself to other humans, developing, in the process, an increasing ability to adapt to its hosts [25]. Many infections have been caused by coronaviruses that affect the respiratory system. Their degree of severity varies from mild (like the common cold) to dangerous (like MERS and SARS and the more recent COVID-19) [26]. As a rule, the severity of the effects triggered by a virus in a body largely rests on the capability of the body itself (and its immune system) to counteract the virus; fortunately, the immune system is able to overcome it more often than not, either by neutralizing or eliminating it [27]. Another denomination for COVID-19 (acronym for coronavirus disease 2019) is acute respiratory disease from SARS-CoV-2, since it is an infectious disease caused by the SARS-CoV-2 virus that affects the respiratory system. Although official reports place the start of its spread in December 2019 from the Chinese province of Huan, doubts have been raised by some experts in the field, who consider it should be placed, instead, in an earlier period of time. COVID-19 was officially classified as the first coronavirus pandemic on 11 March 2020, by the WHO, the World Health Organization [28]. An assessment backed by data, is that in the span of three months since the first verified cases, the virus had spread across 210 countries, infecting by 23 May of the same year more than 5,347,886 people and causing the death of 340,879 [29]. Since the virus is relatively new, our bodies are not yet equipped to fight it off, and it will take vaccination campaigns months before they can provide enough coverage to stop the virus from spreading. What makes this pandemic especially worrying is the rapid speed rate at which the COVID-19 virus spreads, and its mortality rate: The WHO estimated it to be around 3-4\% [30]. The major issue caused by such an exponential contagion is, unavoidably, overloaded health facilities: Their bed availability (or lack thereof) is a critical need not only for present and future COVID-19 patients, but also for the rest of the patients affected by unrelated pathologies. Until an effective mass vaccination campaign grants the population widespread immunity from the virus, public health measures to reduce the base $\mathrm{R}_{0}$ reproduction number (hence limiting new infections) have to be taken. The $R_{0}$ parameter measures the number of people, on average, that an infected person can transmit the disease to, in a population that has not yet encountered the new virus, and hence cannot possess any kind of immunity to it. It is therefore a numerical measure of the potential transmissibility of infectious diseases. As a general rule, our capability to contain the pandemic is inversely proportional to the $R_{0}$, with a threshold value of 1 : Therefore, a $R_{0}>1$ signifies high contagion rate, whereas $\mathrm{R}_{0}<1$ indicates its decline and, eventually, its extinction. According to the $\mathrm{WHO}$, the basic reproduction number for SARS-CoV-2 is between 2 and 2.5, which means that 2.25 people are infected per positive person [30]. The significance of the $R_{0}$ parameter lies in its synthesis of three critical variables that concern the infected person: (i) the likelihood they could be able to transmit the virus with a single contact, (ii) the number of people who had direct contact with them, and (iii) the length of time they remain contagious. Decreasing the number of any of these three values would allow for an increased chance of delaying, or even limiting, the spread of the virus. 


\section{Blockchain Technology: Current and Future Uses}

The Bitcoin blockchain [1] is a public distributed ledger that is free of any corporate or other influence, and is open to anyone willing to use it. It was created to record transactions of the digital cryptocurrency Bitcoin. In this section, we briefly introduce and explain this technology and shortly review its most relevant characteristics, which will allow for a better understanding of how we intend to employ them in our software architecture application [31,32].

In the Bitcoin economy, a fixed number of new bitcoins are minted every 10 minutes on average. Informally speaking, bitcoins are always tied to Bitcoin addresses (from the moment they are minted) and they can be transferred from one address to another by means of transactions. Anyone can generate new addresses, receive a quantity of bitcoins, and send them to other addresses. In theory, anyone is also allowed to participate in the race to generate new bitcoins. There is no authority regulating the minting process ("mining" in the language of Bitcoin) and no bank managing transactions. All this is possible thanks to an ingenious combination of a few classical cryptographic concepts (namely, cryptographic hash functions [33] and digital signature schemes [34] with the flexibility of peer-to-peer networks [35].

All nodes participating in the Bitcoin system are connected to an unstructured peer-topeer network running on top of the Internet: Every time the Bitcoin software is executed on a computer (or smartphone, tablet, or any other device with the appropriate computational and connectivity capabilities), that computer becomes a node in the network. The other nodes in the network bring the new one up to date with the latest system state by sharing with it a stream of packets of information. Each and every packet received by a node from its neighbors contains the necessary information to verify that everything is in order with the packet itself. Only if under this scrutiny it proves to be legitimate is it is sent by the node to all the other nodes in the network. All of them then run the very same procedure so that in the end, each and every node has sent the verified packet to all of its neighbors. The entire procedure takes about two seconds to complete. Within the Bitcoin network, in most cases the exchanged packets contain either a block or a transaction.

Simply put, the elements that make a transaction are input and output addresses, whose number varies according to necessity, digital signatures, and room to eventually store a message. When a transaction takes place the input addresses of the bitcoins being transferred are assigned to their output addresses. To provide a means to guarantee the validity of the input addresses, they are assigned private keys that are mandatory to the creation of digital signatures. As mentioned, it takes a couple seconds or so for a packet or (in the case of Bitcoin) for a transaction sent by a node to reach all of its neighbors (or other nodes) in the network. Its successful reception alone, however, does not suffice to deem the transaction as verified, although it is true that at this point every node knows the input address from which the transaction was sent, and hence are able to recognize any transaction coming from the same address as invalid. Instead, a transaction can be verified when a miner includes it in the next valid block.

Some nodes, called miners, stand out from the others by doing additional operations. In fact, not only do they perform standard ones, but they also attempt to add to the chain of blocks that constitutes the ledger, the next valid set of records. Creating it requires computing devices capable of handling extremely complex calculations. The effort of miners is amply rewarded, should they succeed: Not only they are given the newly created bitcoins, but they also earn the transaction fees of any transaction they manage to include in the block. A block can be considered valid if and only if it contains (i) a link to the previous valid block; (ii) a timestamp, a digital fingerprint of the set of transactions included in the block; and (iii) a nonce, i.e., a number that satisfies some properties. Finding such a nonce is a matter of brute-force search, and the chances that a specific miner finds one are directly proportional to the computational power the miner uses for that purpose. As soon as a miner finds such a nonce for its own block, it sends the block to all nodes via the peer-to-peer network. Each node can independently verify that the block is actually valid, 
and all miners will start considering that one as the last valid block to link. The sequence of blocks, each one linked to the previous one, up to the genesis block mined by Satoshi Nakamoto in January 2009, forms the blockchain [36].

\section{How Blockchain Can Be Used to Support the Emergence}

Technological progress is one of the key factors that can help to overcome the challenges posed by the COVID-19 pandemic. New technologies, such as the Internet of Things (IoT), artificial intelligence (AI), machine learning (ML), blockchain, robotics, unmanned aerial vehicles (UAVs), three-dimensional printing, nanotechnology and synthetic biology, 5G communications, cloud and edge computing, and big data, can be leveraged to develop efficient emergency management strategies for the COVID-19 pandemic. Moreover, blockchain technology has been identified by the European Parliamentary Research Service as one of the 10 key technologies to fight COVID-19 [37]. The trusted and reliable nature of this architecture makes it suitable to fight the pandemic. Indeed, in every human interaction, trust must be in place because it is not a behavior (e.g., cooperation) or a choice (e.g., taking a risk), but a primary psychological condition that can cause or result from such actions [38]. Moreover, it is not transitive [39]. Distributed ledger technology allows participants to trust the outcome of a system without trusting the individual participants, which is a precondition for economic efficiency and prosperity [40-42].

The new coronavirus created specific issues. Protective equipment and large amounts of money are being donated by people and communities. However, a common concern is that donations are not always being used where needed and that supplies are being misdirected. There are many reasons behind these problems, but they impact the willingness of people to donate and thus delay the objective of addressing the problem.

\subsection{Monetary Donation}

The exponential growth of infected people together with the need to intubate and assist patients in intensive care for long periods led hospitals in Italy to be under enormous pressure, and to manage this new and dangerous scenario, several fundraisers have been created. Monetary donations are a very complex topic, as people are often unable to decide between the desire to offer money and the feeling of skepticism or lack of trust for the actual use of the sum offered. To overcome this problem, we suggest using blockchain technology. This technology is already in widespread use, so why is it not being used for charity as well?

As an example, think about an association that decides to open a fundraiser for a specific hospital and that to do so, chooses to rely on a blockchain platform. The association will be able to create a public digital portfolio associated with the project. By publicly associating a Bitcoin address (which can be compared to an IBAN bank code) to a specific project, anyone can observe the transfers of money in real time in the blockchain. This happens because all the transactions that take place within the Bitcoin network are recorded in the blockchain. In this way, all network participants will be enabled to proceed with the donation. Once the money is received, the association will be able to transfer it to the hospital by entering the related documentation on the platform. The use of blockchain offers the possibility for all stakeholders, donors, and donees to follow, and therefore verify, every monetary transfer, a mechanism also reinforced by the insertion of the related documentation [43]. The transparency guaranteed by this system would therefore solve the problem related to the skepticism previously exposed, thus allowing the number of donations to increase and funds to be more quickly collected and transferred to hospitals, charities, or other initiatives (see Figure 1). 

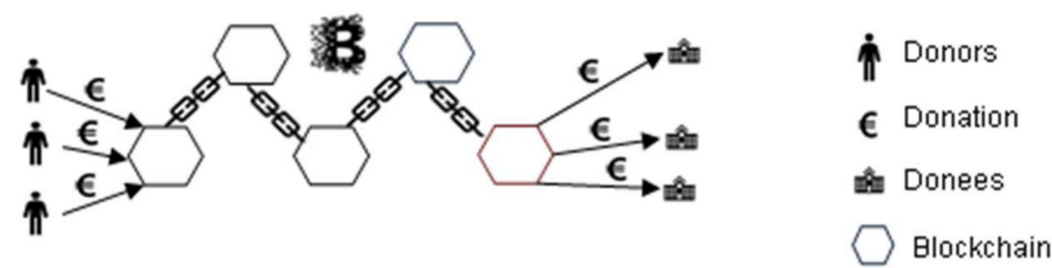

Figure 1. Monetary donation flow.

\subsection{Technical and Sanitary Material Donations}

Due to the ongoing COVID-19 pandemic, the world has witnessed profound supplychain disruptions. Industrial production activities are at a standstill on the one hand because lockdowns have been imposed, and on the other hand, because factories are not equipped and/or designed to follow the new paradigm of social distancing and working with physical distancing. Furthermore, import and export bans have affected the global supply chain. Even though at present it is impossible to understand the precise level of disruption the pandemic has caused in the global supply chain, it is obvious that it has caused serious crises in supply and demand. The type of goods required can cause either high demand or high supply. Medical equipment and pharmaceutical supply chains have found, and still are finding, difficulties in keeping the entire chain intact and meeting the higher demand [11].

In a difficult moment such as the one experienced by a country during the SARSCoV-2 pandemic, manufacturing companies and some foreign countries have offered to donate technical and/or sanitary materials to hospitals. For different reasons, the products offered may not arrive at their proper destination. As an example, think of a manufacturing company that has been able to convert its primary production in order to donate masks and protective suits to a hospital and that the transport of the products takes place by air.

Blockchain can be used to trace the entire path (see Figure 2). At any access point in the system, the presence of a trusted person capable of reporting the transaction on the blockchain should be guaranteed; they should also include, when possible, the dematerialized documents such as the bill of lading or the receipt signature. The trusted actors are the manufacturing company, the airport surveillance, the transport companies for each dispatch and receipt point of the product, and the hospital. Leaving the factory, the products are loaded onto an initial transport vehicle and an authorized person adds the transaction to the chain (i.e., the presence of the materials on the vehicle); then a second person reports the arrival of the products to the airport and consequently their boarding onto the plane. This procedure is carried out by other employees, both when the plane has landed and when the products are transferred from the airport to the hospital.

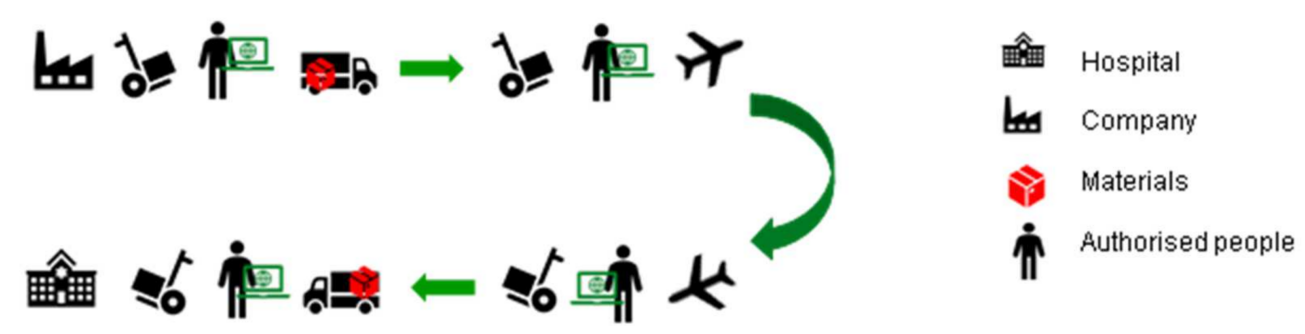

Figure 2. The technical and sanitary material donation flow.

Blockchain technology can play a key role in building a more resilient supply chain. Blockchain can anonymously knit together all stakeholders, establishing a reliable environment. Immutable recording of data logs supports auditability, provenance, and transparency, also providing a high level of automation. 


\subsection{Notification Flow of Suspected COVID-19 Cases}

In Italy, each region received indications regarding the communication flow of suspected COVID-19 cases. The doctor who identifies a suspected case fills out a form-Form for reporting cases of insertion from respiratory viruses - containing the patient's personal and epidemiological clinical information, which refers to the places visited, means of transport used, contacts, etc. The form must then be communicated, via email or phone, to the Prevention Department, and to the corresponding Local Health Authority (Azienda Sanitaria Locale-ASL). In particular, the former uses the information to carry out epidemiological investigations in order to identify all the person's contacts. The LHA sends the document to the Regional Epidemiological Service, which in turn, through a dedicated platform, communicates the data to the Ministry of Health and to the Higher Institute of Health (Istituto Superiore della Sanità-ISS). The latter generally uses this information to monitor the spread of the pandemic nationwide. In the meantime, the samples from the SARS-CoV-2 tests are sent to the regional reference laboratory. When the test results are ready, the doctor updates and transmits the information, again following the notification flow just described [44]. The communications between doctor-LHA and LHA-Regional Epidemiological Service can take place via email or phone: The former is an insecure communication system, easily subject to hacking phenomena, whereas the latter does not even offer the possibility to visualize and preserve the received information (see Figure 3).

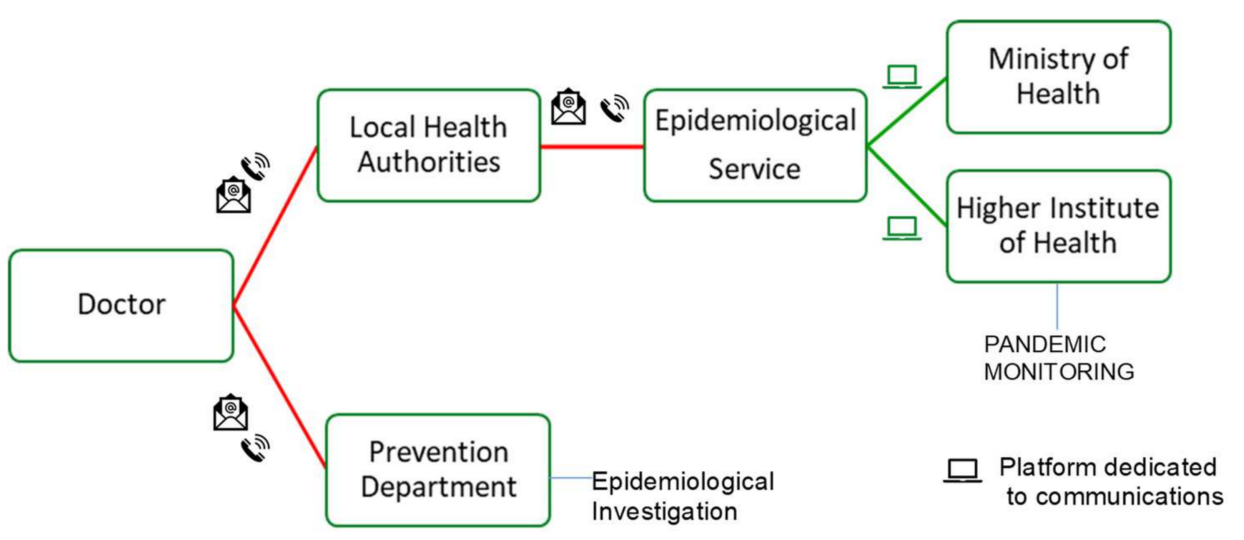

Figure 3. Notification flow of suspected COVID-19 cases.

In order to overcome these problems, our idea is to use blockchain to manage and improve the information exchange just described. In particular, we suggest integrating blockchain with each hospital in the region, which would therefore involve the doctors in the emergency and infectious diseases departments of the hospital, the health personnel of selected wards, LHA officials, the officials of the Regional Epidemiological Service, and the officials from the Prevention Department. Doctors would have the role of inserting the forms and their updates in the blockchain, and of validating their contents. The information would be then part of a block in the blockchain, meaning all selected users would receive an updated copy of it (see Figure 4). 


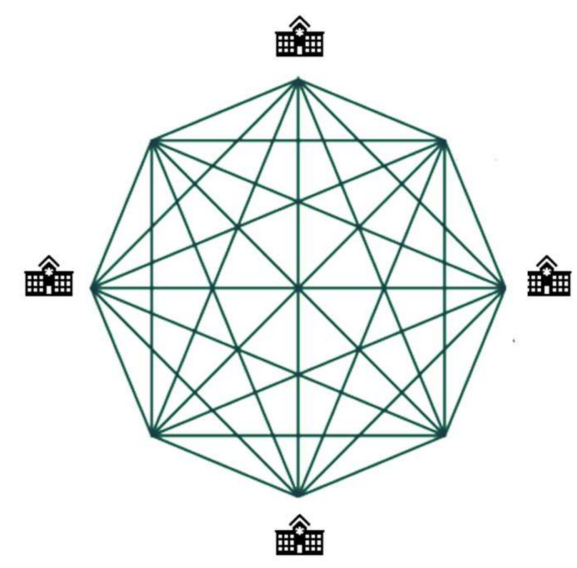

- Doctors

轓 Local Health Authority

Regional Epidemiological Service

Prevention Department

Figure 4. Graphical representation of the proposed model to manage and improve notification flow of suspected COVID-19 cases during the COVID-19 pandemic by using blockchain technology.

The process, structured in this way, can bring several advantages:

i. The notifications of suspected cases and updates would arrive in a safer and timelier way than traditional methods, thus offering the possibility for the health structures involved to anticipate the activities they carry out;

ii. Specialists, nurses, and doctors from the involved wards could consult the data collected by the blockchain in real time in order to work together, sharing ideas and solutions related to the choice of therapies;

iii. The insertion of data in the blockchain would prevent healthcare professionals from spending precious time dealing with the document archiving on the one hand, and on the other, would prevent the archives from becoming overloaded;

iv. In the near future, data from the blockchain could be exploited by the infectious disease specialists as a large COVID-19 clinical research case study.

In the management of the COVID-19 pandemic, data sharing among healthcare collaborators is of crucial importance, since it would help to collect significant data sets, which can play an important role in COVID-19 research. These data-sharing mechanisms must take into account the national data-sharing laws in order to avoid violating them. Indeed, patients' privacy is of great importance and very often is one of the obstacles in the implementation of a medical data-sharing system as well as in its extensive adoption. National authorities enforce robust controls and define data access control policies. Moreover, detailed patient information, such as blood oxygen level, heart rates, and medication doses, can be gathered by integrating it into Medical IoT (MIoT) devices. Although many steps forward have been made to guarantee privacy at a legal and regulatory level, the decentralized nature of blockchain seems to be the right choice to greatly improve the security and privacy of healthcare data. Moreover, patients and hospitals can increase their control over personal data thanks to the removal of costly middleman necessary in centralized databases. Blockchain has all the potential to break the traditional ways of recording medical data and make the data-sharing process between hospitals and medical professionals across the country (and eventually around the world) significantly easier, safer, and faster. A blockchain-based system of real-time data sharing can remove problems such as data counterfeiting and alteration. The consequent transparency in collecting, storing, and sharing data helps to build and maintain trust between stakeholders and protects patients' privacy [11].

\section{The Proposed High-Level Architecture}

As long as the COVID-19 pandemic continues, the need to manage donations of money and technical and sanitary material, as well as the notification flow regarding suspected COVID-19 cases also continues. This information is crucial to allowing all the trusted 
people involved to safely and securely verify the efficient management of the emergency and all the events correlated to it.

The management of all this information and data at a larger scale (thousands of records) requires a novel approach. Blockchain is the perfect tool to store encrypted data, since it guarantees the inalterability of every transaction. Hence, we propose its utilization under the supervision of the authorities, in order to assist with decentralized data recording (and storing) in every step of the process.

It would be necessary for each of these steps to have a unique ID assigned and stored in indivisible conjunction with the identity of trusted people, the data of the transaction, and the transaction timestamp. The result of the process is therefore accessible to all people and all the authorities to verify and monitor what is happening.

The envisioned architecture should possess the following features:

1. A way to assign and store to every step of the process and a unique ID together with the identity of the trusted people involved;

2. A blockchain to enable a safe, encrypted, and unalterable way to store transactions;

3. A software system based on a combination of web services and apps to access the blockchain and verify the information;

4. A digital service or application that allows delegated authorities to monitor and verify the flow of information and materials.

It is worth noting that this method could continue to be used to manage all of these transactions even after the end of the pandemic emergency.

In Figure 5 our high-level architecture is presented.
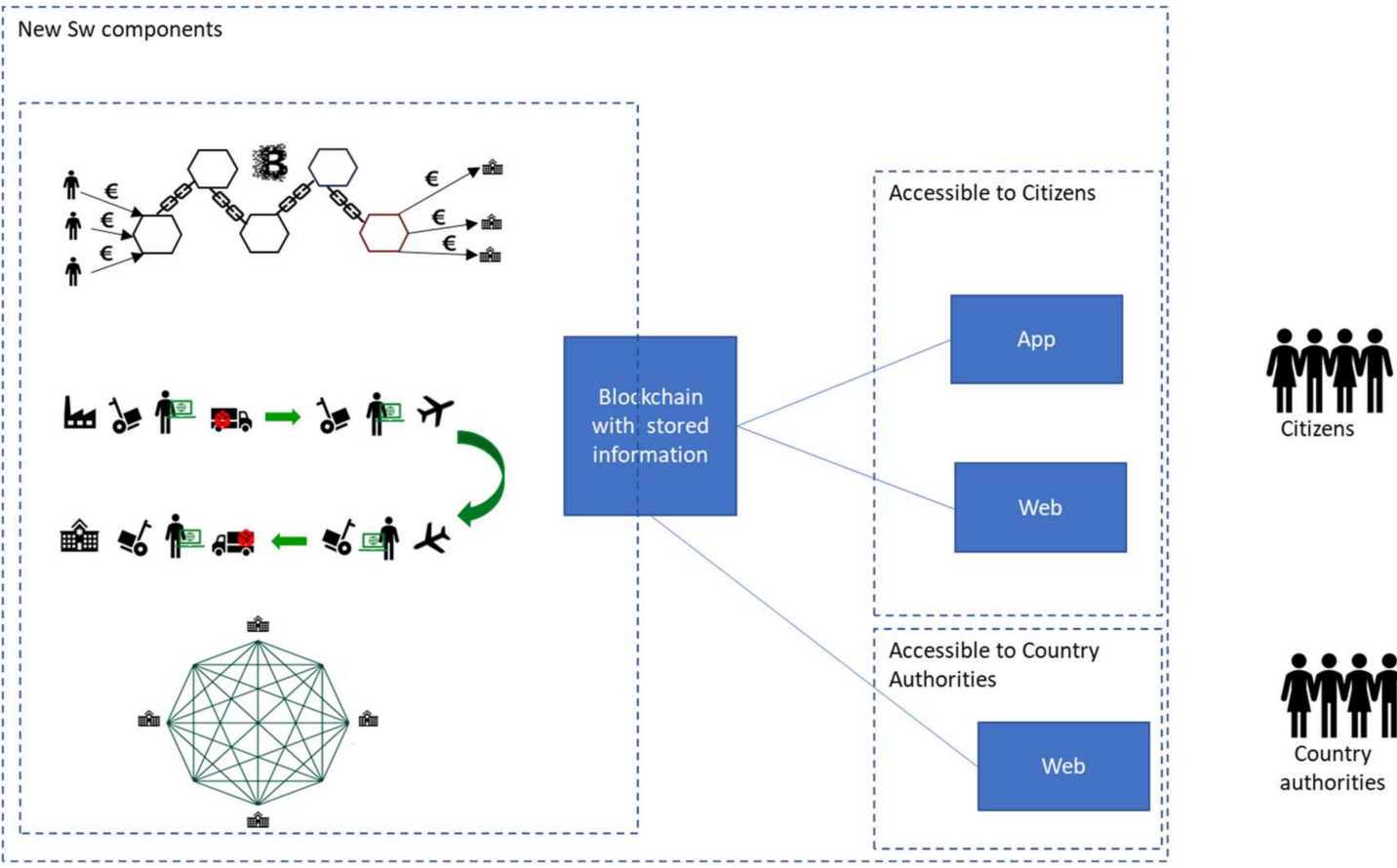

Figure 5. The proposed high-level architecture.

\section{Conclusions}

In this paper, blockchain technology is used in three different scenarios to support COVID-19 pandemic management: (1) notification flows between healthcare infrastructures, and donations of (2) monetary and (3) technical and sanitary materials. The three analyzed scenarios have a common strategic factor: time. The utilization of blockchain can help in the optimization of the response time during an emergency situation. In fact, to counter the spread of a virus it is necessary to act faster than its spread capability. The faster the confirmed cases are identified, the sooner contacts can be located to interrupt 
the virus transmission chain. In the same way, the faster the necessary technical and sanitary materials reach the hospitals, the sooner it will be possible to hospitalize, treat, and discharge patients.

As regards monetary donations, the blockchain used to track them offers the possibility to track money transfers. This mechanism solves the problem of lack of trust linked to the uncertainty about the real use of the money offered, thus allowing the number of donations to hospital and healthcare infrastructures to increase.

As regards technical and sanitary material donations, blockchain allows for the tracking of the products in order to facilitate the identification of the causes of any drawbacks such as, for example, product loss, thus increasing the probability that the materials arrive at hospitals in the established times and ways. The need for trusted people in the system's access points inevitably leads to weakening the potential of the use of blockchain. For this reason, we are not talking about the complete abolition of unexpected events, but rather a simplification in identifying their causes.

Blockchain, when applied to the flow of information between health infrastructures, allows the timing of the communications to be accelerated. Unfortunately, many national health systems lack the most modern forms of digitization. This pandemic has highlighted these shortcomings and stressed the need to digitize hospital and healthcare management processes.

Our paper aims to propose a digital solution to help manage the phase of the pandemic when the need to monitor what is happening emerges as a priority and when it is crucial for the information to be verified and tamper-proof. Our proposal aims to assist in administrating large-scale information and storing the gathered data in a distributed way, in a blockchain supervised and overseen by the authorities.

Once the pandemic is over, the governments and health authorities of countries worldwide must study new pandemic plans, following the example of South Korea. In future projects, they cannot miss digital and innovative support systems such as blockchain, which represents a valid tool in support of the health system. Our hope is that such a scenario will not be repeated, but if a new pandemic wave appears again, it will be necessary to be better prepared and use all the technological means aimed at stopping the spread of new epidemic and all the related problems.

\section{Discussion and Limitation}

During the last few years, the utilization of blockchain technology has become a hot topic due to its undeniable potential. However, it would be a mistake to think that this technology can be easily and immediately applied, because blockchain is a very complex technology and few people have begun to explore all its potential. Blockchain is not a solution that will fix everything that is not efficiently managed nowadays. However, it does offer new ways to improve the management of different systems we have today, and that is the reason why it is so interesting and challenging to explore. Blockchain was born as a disruptive technology for digital currency, and now it is showing itself to be an open resource with possibilities in different fields. The key to the interest in this technology lies in its distributed system that ensures no alteration of the information and the maintenance of privacy. Every institution and enterprise capable of exploiting these technologies will have a chance to radically improve and/or change existing processes, create new business models, and develop innovative products and services able to answer the different needs of a new generation of consumers that is increasingly demanding and aware. Until a few years ago, what was a utopian and tech-enabled vision of the future is available today: blockchain, a technology able to keep an unalterable record of every exchange, removing the need for trusted, third-party intermediaries in digital transactions.

Gartner estimates that blockchain could create USD 176 billion of value-added revenue by 2025 , revolutionizing the supply chain, enabling new business models, and disrupting existing ones. In fact, the consequences of the utilization and the complete exploitation of this technology are faster processes, real-time transaction visibility, and reduced costs across every industrial, social, and economic sector. 
In our paper, the management of the emergency situation of the COVID-19 pandemic was taken into consideration. Such an emergency was chosen precisely because it is the leading situation in which the use of appropriate technologies and the digitization of information can really make a difference and save many lives.

We have to highlight the apparent inability of IT systems (lacking completely in some cases) to support the processes of the management and storage of data during an emergency period. There is still insufficient knowledge of the most advanced technological tools and little awareness of the great difference they could make in the management of crisis or emergency situations. This paper proposes a solution to address these problems in order to improve the management of such situations, which is now so inefficient that it appears to have lost the trust of the people. However, it is indispensable to understand the complexities of this innovative solution, which, being still under development, causes a marked divergence of opinions over whether to adopt it or not. Unfortunately, often when such radical changes are introduced it is difficult to get them accepted and therefore there is great resistance to accepting them, even if they would bring numerous advantages.

The solution proposed in this paper guarantees transparency and especially immutability, which are essential for secure management and storage in critical emergency situations, ensuring a system that is efficient and, hopefully, brings renewed trust.

Realizing the full potential of such a system would require collaboration and standardization between parties that are different in nature, but share convergent needs and interests: the efficient and timely management of the emergency. Moreover, the introduction of blockchain technology to address these problems and the considerable costs they would entail might make one wonder whether blockchain is not just another example of an inadequate innovation. In this case, the best strategy would be to introduce this technology gradually: Tests and analyses will determine the path to follow to ensure sufficient returns on the technological investment step-by-step. It should therefore be emphasized that the aim of this paper is to lay the groundwork for more in-depth study in the future, which would include cost-benefit analysis and an evaluation of indicators such as ROI to determine the feasibility of the project. This paper simply proposes an architecture in which all the implications of its IT implementation should be the subject of further study.

Before the creation of blockchain technology, no one would have ever thought of using interoperable trust architecture in the management of crisis and emergency situations. Blockchain technology offers the possibility to realize such a system, precisely because it differs from past solutions in its capacity to simplify and speed up the exchange of data through an unalterable, decentralized architecture, creating a secure, immutable, shared ledger. Blockchain technology can actually act as a catalyst for the innovation of services offered to and for citizens. Politics must play an active role that fully understands the fundamental role of innovation in every sector and mainly in an emergency period such as the COVID-19 one, encouraging the digital culture and related infrastructures. In fact, when several actors are involved in a transaction, as in the case of the services offered during an emergency situation, the need for transparency, speed, accuracy, and security increase. Meeting those demands while maintaining high levels of quality is a key factor for governments in order to create value for citizens as well as to increase the trust of the latter in a historical moment in which trust in public institutions is reduced to minimum terms. The attention of national governments will have to be directed toward the implementation of a development and growth strategy involving multiple decisionmaking levels (companies, universities, citizens, startups, research centers, etc.) and place a more participatory style of governance of local systems at the center. Therefore, solid digital knowledge is needed to take advantage of the opportunities offered by new technologies such as blockchain, artificial intelligence, or the Internet of Things, to build a solid computer-based culture on a continuous and reliable interaction with citizens, businesses, and national public administrations.

The application of blockchain technology is clearly a complex and gradual process that must also face obstacles such as people's low level of digital knowledge and pre-existing 
paper-based processes, where all the involved stakeholders (including citizens) expect to receive a physical document printed on a sheet of paper as a result of the process. The delay in the technological transformation is also of a psychological nature, as people often find it difficult to deal with a change, even if necessary or helpful. Feelings like discomfort and uncertainty arise every time people face the management of an innovation in terms of something that differs from what is usual and familiar; nevertheless, the aversion to change should not delay the unavoidable process of digitization and innovation. Blockchain technology has the capacity to greatly expand the possible boundaries and scenarios of the development of emergency management functions: Once an adequate level of technological maturity is reached, decentralization in the administration will be one natural, expected, and revolutionary consequence of the application of this technology. Therefore, it is crucial to start acquiring the necessary awareness to push governments to take action in this direction and to carry out the fundamental changes all citizens need to gain confidence with the new technologies.

In conclusion, the potential of blockchain technology is huge and the consequent benefits are too important not to be taken into consideration.

This study is in an initial stage and for this reason has some limitations. It has a more qualitative than quantitative approach, but we are conducting an exploratory analysis and we aim to continue our studies of the application of blockchain in the different sectors, trying to contribute to the exploitation of a standard and open-source framework for monitoring and auditing electronic records. Blockchain is undoubtedly transformative and, for this reason, much of its impact has yet to be explored, even on a theoretical level. In our future research we aim to evaluate how the efficient management of an emergency or crisis period could accommodate such innovation in terms of digital divide and we also aim to implement a cost model suitable for the sectors involved in such periods. Our goal is not mere economic savings, but the efficiency and scalability of the process in terms of time, which would affect not only the process itself, but also other processes that could gain more time and resources. Moreover, time also plays a crucial role when dealing with peoples' safety in an emergency situation.

Author Contributions: Both the authors contributed to the developing and writing of the manuscript. All authors have read and agreed to the published version of the manuscript.

Funding: This research received no external funding.

Institutional Review Board Statement: Not applicable.

Informed Consent Statement: Not applicable.

Data Availability Statement: Not applicable.

Conflicts of Interest: The authors declare no conflict of interest.

\section{References}

1. Nakamoto, S. Bitcoin: A Peer-to-Peer Electronic Cash System. 2008. Available online: http://bitcoin.org/bitcoin.pdf (accessed on 13 July 2021).

2. Catalini, C.; Gans, J.S. Some Simple Economics of the Blockchain. Rotman School of Management Working Paper No. 2874598; MIT Sloan Research Paper No. 5191-16. 2017. Available online: https:/ /ssrn.com/abstract=2874598 (accessed on 16 November 2020).

3. Gupta, V. The Promise of Blockchain Is a World Without Middlemen. Harvard Bus. Rev. 2017. Available online: https: / /hbr.org/2017/03/the-promise-ofblockchain-is-a-world-without-middlemen? (accessed on 16 November 2020).

4. Giungato, P.; Rana, R.; Tarabella, A.; Tricase, C. Current Trends in Sustainability of Bitcoins and Related Blockchain Technology. Sustainability 2017, 9, 2214. [CrossRef]

5. Vranken, H. Sustainability of bitcoin and blockchains. Curr. Opin. Environ. Sustain. 2017, 28, 1-9. [CrossRef]

6. Beck, R.; Müller-Bloch, C. Blockchain as Radical Innovation: A Framework for Engaging with Distributed Ledgers. In Proceedings of the 50th Hawaii International Conference on System Sciences; 2017. Available online: https://www.researchgate. net/publication/312166392_Blockchain_as_Radical_Innovation_A_Framework_for_Engaging_with_Distributed_Ledgers_as_ Incumbent_Organization (accessed on 3 June 2020).

7. Allen, D. Discovering and Developing the Blockchain Cryptoeconomy. 2017. Available online: https://ssrn.com/abstract=2815 255 (accessed on 20 October 2020). 
8. Tapscott, D.; Tapscott, A. The Blockchain Revolution and Higher Education. Educ. Rev. 2017, 52, 11-24. Available online: https:/ / er.educause.edu/articles/2017/3/the-blockchain-revolution-and-higher-education (accessed on 20 October 2020).

9. Bansal, A.; Garg, C.; Padappayil, R.P. Optimizing the implementation of COVID-19 "immunity certificates" using blockchain. J. Med. Syst. 2020, 44, 1-2. Available online: https://link.springer.com/article/10.1007\%2Fs10916-020-01616-4 (accessed on 13 February 2021).

10. Hasan, H.R.; Salah, K.; Jayaraman, R.; Arshad, J.; Yaqoob, I.; Omar, M.; Ellahham, S. Blockchainbased solution for COVID-19 digital medical passports and immunity certificates. TechRxiv 2020. [CrossRef]

11. Kalla, A.; Hewa, T.; Mishra, R.A.; Ylianttila, M.; Liyanage, M. The role of blockchain to fight against COVID-19. IEEE Eng. Manag. Rev. 2020. Available online: https://ieeexplore.ieee.org/document/9157910 (accessed on 14 February 2020).

12. Marbouh, J.D.; Abbasi, T.; Maasmi, F.; Omar, I.; Debe, M.; Salah, K.; Jayaraman, R.; Ellahham, S. Blockchain for COVID-19: Review, opportunities and a trusted tracking system. TechRxiv. Preprint 2020. [CrossRef]

13. Rao, R.; Jain, H. Improving Integrated Clinical Trial Management Systems through Blockchain. Genetic Engineering \& Biotechnology News, 19 November 2019. Available online: https://www.genengnews.com/insights/improving-integrated-clinical-trialmanagement-systems-through-blockchain/(accessed on 28 May 2020).

14. Glover, D.G.; Hermans, J. Improving the Traceability of the Clinical Trial Supply Chain. App. Clin. Trials 2017, 36-38. Available online: https:/ / www.appliedclinicaltrialsonline.com/view/improving-traceability-clinical-trial-supply-chain (accessed on 20 May 2020).

15. Nugent, T.; Upton, D.; Cimpoesu, M. Improving data transparency in clinical trials using blockchain smart contracts. F1000Research 2017, 5, 1-4. Available online: https:/ / pubmed.ncbi.nlm.nih.gov/28357041/ (accessed on 30 May 2020). [CrossRef] [PubMed]

16. Dragov, R.; Croce, C.L.; Hefny, M. How Blockchain Can Help in the COVID-19 Crisis and Recovery. IDC-Analyze the Future, 4 May 2020. Available online: https://blog-idcuk.com/blockchain-help-in-the-covid-19-and-recovery/(accessed on 3 June 2020).

17. Blockchain And Crypto Firm VeChain Utilized to Confirm Authenticity of Coronavirus KN95 Masks. Blockchain Magazine, 20 April 2020. Available online: https:/ / www.blockchainmagazine.net/blockchain-and-crypto-firm-vechain-utilized-to-confirmauthenticity-of-coronavirus-kn95-masks / (accessed on 30 May 2020).

18. Singh, G.; Levi, J. MiPasa project and IBM Blockchain team on open data platform to support Covid-19 response. IBM, 27 March 2020. Available online: https://www.ibm.com/blogs/blockchain/2020/03/mipasa-project-and-ibm-blockchain-team-on-opendata-platform-to-support-covid-19-response/(accessed on 30 May 2020).

19. Nguyen Q., V.; Cao, D.A.; Nghiem, S.H. Spread of COVID-19 and policy responses in Vietnam: An overview. Int. J. Infect. Dis. 2021, 103, 157-161. Available online: https:/ / www.sciencedirect.com/science/article/pii/S1201971220324668 (accessed on 14 April 2021). [CrossRef] [PubMed]

20. Coalition: Help Stop the Spread. Available online: https://www.coalitionnetwork.org/ (accessed on 30 June 2020).

21. Joshi, M. PHBC announces blockchain monitor to track virus-free zones. Cryptopolitan 2020. Available online: https://www. cryptopolitan.com/phbc-blockchain-monitor-for-virus-free-zones/ (accessed on 27 May 2020).

22. Chang, M.C.; Park, D. How can blockchain help people in the event of pandemics such as the COVID-19? J. Med. Syst. 2020, 44, 1-2. [CrossRef] [PubMed]

23. Zhang, J. Chinese startup launches blockchain platform to improve donation efficiency. Tech Asia 2020. Available online: https://www.techinasia.com/china-blockchain-platform-donation (accessed on 27 May 2020).

24. Acoer. Providing Daily Tracking and Visualizations of the Pandemic. Coronavirus (COVID-19) Tracker. Available online: https: / / www.acoer.com/coronavirus (accessed on 27 May 2020).

25. Clementi, N. Coronavirus Cinese: Cosa Sappiamo Sull'origine. 2019. Available online: https://www.medicalfacts.it/2020/01/23 / coronavirus-cinese-italia/ (accessed on 30 August 2020).

26. EpiCentro-The Portal of Epidemiology for Public Health by the Italian Higher Institute of Health (ISS). "Malattie Infettive". 2020. Available online: https:/ / www.epicentro.iss.it/infettive/sorveglianza (accessed on 30 January 2020).

27. Italian Ministry of Health. FAQ_Covid-19, Domande e Risposte. 2020. Available online: http:/ /www.salute.gov.it/portale/ malattieInfettive/dettaglioFaqMalattieInfettive.jsp?lingua=italiano\&id=228 (accessed on 30 January 2020).

28. Italian Ministry of Health. L'Organizzazione Mondiale Della Sanità Dichiara il Coronavirus Pandemia. 2020. Available online: http:/ / www.salute.gov.it/portale/nuovocoronavirus/dettaglioNotizieNuovoCoronavirus.jsp?lingua=italiano\&menu= notizie \& $\mathrm{p}=$ dalministero\&id $=4209$ (accessed on 13 July 2020).

29. Worldometer. Covid-19 Coronavirus Pandemic. 2020. Available online: https://www.worldometers.info/coronavirus/ (accessed on 13 July 2020).

30. World Health Organization. Q\&A: Similarities and Differences-COVID-19 and Influenza. 2020. Available online: https: / / www.who.int/emergencies / diseases / novel-coronavirus-2019/question-and-answers-hub / q-a-detail/q-a-similarities-anddifferences-covid-19-and-influenza (accessed on 21 July 2020).

31. Narayanan, A.; Bonneau, J.; Felten, E.; Miller, A.; Goldfeder, S. Bitcoin and Cryptocurrency Technologies: A Comprehensive Introduction; Princeton University Press: Princeton, NJ, USA, 2016.

32. Antonopoulos, A.M. Mastering Bitcoin: Programming the Open Blockchain; O'Reilly Media, Inc: Sebastopol, CA, USA, 2017.

33. Damgård, I.B. Collision Free Hash Functions and Public Key Signature Schemes. In Advances in Cryptology—EUROCRYPT' 87; Springer: Berlin/Heidelberg, Germany, 1988; Volume 304, pp. 203-216.

34. Diffie, W.; Hellman, M. New directions in cryptography. IEEE Trans.Inform. Theory 1976, 22, 644-654. [CrossRef] 
35. Barkai, D. Peer-to-Peer Computing: Technologies for Sharing and Collaborating on the Net. Intel Press: Santa Clara, CA, USA, 2001.

36. Capece, G.; Bazzica, P. V-passport: Vpassport: A Digital Architecture to Support Social Restart during the SARS-CoV-2 Pandemic. Sustainability 2021, 13, 3945. [CrossRef]

37. Kritikos, M. Ten Technologies to Fight Coronavirus. 2020. Available online: http://www.europarl.europa.eu/RegData/etudes/ IDAN/2020/641543/EPRS_IDA (accessed on 5 March 2021).

38. Rousseau, D.; Sitkin, S.; Burt, R.; Camerer, C. Not So Diffeerent After All: A Cross-discipline View of Trust. Acad. Manag. Rev. 1998, 23, 393-404. [CrossRef]

39. Werbach, K. Trust, But Verify: Why the Blockchain Needs the Law. Berkeley Technol. Law J. 2018, 33, 487. Available online: https: / / ssrn.com/abstract=2844409 (accessed on 20 October 2020).

40. North, D. Institutions, Institutional Change, and Economic Performance; Cambridge University Press: Cambridge, UK, 1990.

41. Nooteboom, B. Trust: Forms, Foundations, Functions, Failures and Figures; Edward Elgar: Cheltenham, UK, 2002.

42. Davidson, S.; De Filippi, P.; Potts, J. Economics of Blockchain. 2016. Available online: https://ssrn.com/abstract=2744751 (accessed on 20 October 2020).

43. Settembre, D. Storia di Helperbit, la Startup Italiana per le Donazioni in Criptovaluta. 2019. Available online: https:/ forbes.it/ 2019/01/25/fintech-helperbit-donazione-bitcoin-criptovalute/ (accessed on 30 October 2020).

44. Regione Lazio. Indicazioni Operative per la Gestione e la Sorveglianza dei Casi Sospetti di Infezione da Nuovo Coronavirus. 2020. Available online: https:/ /www1.ordinemediciroma.it/images/News/RL_Coronavirus_1.pdf (accessed on 30 October 2020). 